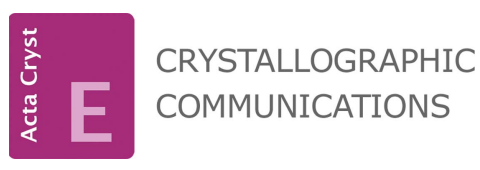

ISSN 2056-9890

Received 24 April 2019

Accepted 21 May 2019

Edited by M. Weil, Vienna University of Technology, Austria

Keywords: crystal structure; pyridazine; Hirshfeld surface analysis.

CCDC reference: 1917654

Supporting information: this article has supporting information at journals.iucr.org/e

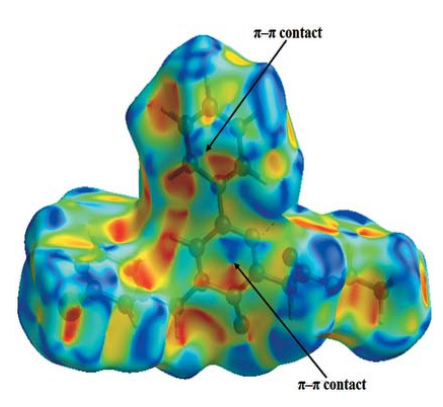

OPEN $\odot$ ACCESS

\section{Crystal structure and Hirshfeld surface analysis of ethyl 2-[5-(3-chlorobenzyl)-6-oxo-3-phenyl-1,6-di- hydropyridazin-1-yl]acetate}

\author{
Fouad El Kalai, ${ }^{a *}$ Cemile Baydere, ${ }^{\mathrm{b} *}$ Said Daoui, ${ }^{\text {a }}$ Rafik Saddik, ${ }^{\mathrm{c}}$ Necmi Dege, ${ }^{\mathrm{b}}$ \\ Khalid Karrouchi ${ }^{\mathrm{d}}$ and Noureddine Benchat ${ }^{\mathrm{a}}$
}

\begin{abstract}
Laboratory of Applied Chemistry and Environment (LCAE), Faculty of Sciences, Mohamed I University, 60000 Oujda Morocco, 'bepartment of Physics, Faculty of Arts and Sciences, Ondokuz Mayıs University, 55139-Samsun, Turkey,

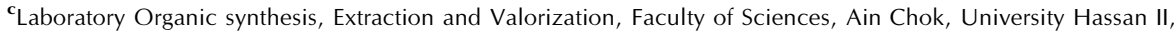
Casablanca, Morocco, and ' Laboratory of Plant Chemistry, Organic and Bioorganic Synthesis, URAC23, Faculty of Science, BP 1014, GEOPAC Research Center, Mohammed V University, Rabat, Morocco. *Correspondence e-mail: fouadelkalai80@gmail.com, cemle28baydere@hotmail.com
\end{abstract}

The title pyridazinone derivative, $\mathrm{C}_{21} \mathrm{H}_{19} \mathrm{ClN}_{2} \mathrm{O}_{3}$, is not planar. The unsubstituted phenyl ring and the pyridazine ring are inclined to each other, making a dihedral angle of $17.41(13)^{\circ}$ whereas the Cl-substituted phenyl ring is nearly orthogonal to the pyridazine ring $\left[88.19(13)^{\circ}\right]$. In the crystal, $\mathrm{C}-\mathrm{H} \cdots \mathrm{O}$ hydrogen bonds generate dimers with $R_{2}^{2}(10)$ and $R_{2}^{2}(24)$ ring motifs which are linked by $\mathrm{C}-\mathrm{H} \cdots \mathrm{O}$ interactions, forming chains extending parallel to the $c$-axis direction. The intermolecular interactions were investigated using Hirshfeld surface analysis and two-dimensional fingerprint plots, revealing that the most significant contributions to the crystal packing are from $\mathrm{H} \cdots \mathrm{H}(44.5 \%), \mathrm{C} \cdots \mathrm{H} /$ $\mathrm{H} \cdots \mathrm{C}(18.5 \%), \mathrm{H} \cdots \mathrm{O} / \mathrm{H} \cdots \mathrm{O}(15.6 \%), \mathrm{Cl} \cdots \mathrm{H} / \mathrm{H} \cdots \mathrm{Cl}(10.6 \%)$ and $\mathrm{C} \cdots \mathrm{C}$ $(2.8 \%)$ contacts.

\section{Chemical context}

Pyridazines are an important family of six-membered aromatic heterocycles (Akhtar et al., 2016). The related compound pyridazinone is an important pharmacophore with a wide range of biological applications (Asif, 2015), and its chemistry has been studied for several decades. Pyridazinones are used as scaffolds for potential drug candidates (Dubey \& Bhosle, 2015; Thakur et al., 2010) because of their significant potential as antimicrobial (Sönmez et al., 2006), antidepressant (Boukharsa et al., 2016), anti-inflammatory (Barberot et al., 2018), antihypertensive (Siddiqui et al., 2011), analgesic (Gökçe et al., 2009), anti-HIV (Livermore et al., 1993), anticonvulsant (Partap et al., 2018; Sharma et al., 2014), cardiotonic (Wang et al., 2008), antihistaminic (Tao et al., 2012), glucan synthase inhibitors (Zhou et al., 2011), phosphodiesterase (PDE) inhibitors (Ochiai et al., 2012) and herbicidal (Asif, 2013) agents.

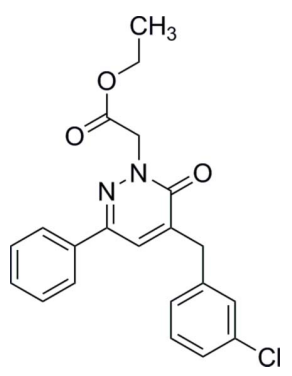




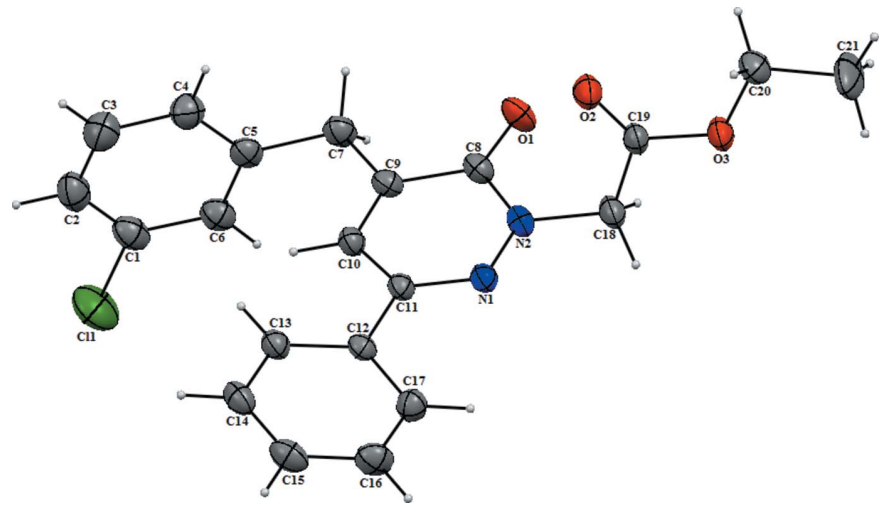

Figure 1

The molecular structure of the title compound with displacement ellipsoids drawn at the $50 \%$ probability level.

In this context and in a continuation of our work in this field (Chkirate et al., 2019a,b; Karrouchi et al., 2015, 2016a,b), we report herein the synthesis and the molecular and crystal structures of the title pyridazinone derivative, together with its Hirshfeld surface analysis.

\section{Structural commentary}

The molecule of the title compound is not planar (Fig. 1). The unsubstituted phenyl ring $(\mathrm{C} 12-\mathrm{C} 17)$ and the pyridazine ring $(\mathrm{C} 8-\mathrm{C} 11 / \mathrm{N} 1 / \mathrm{N} 2)$ are twisted relative to each other, making a dihedral angle of $17.41(13)^{\circ}$; the chloro-substituted phenyl ring (C1-C6) is inclined to the pyridazine ring by $88.19(13)^{\circ}$. Atoms C8 and N2 of the pyridazine ring show the largest deviations from planarity (root-mean-square deviation = $0.0236 \AA$ ) in positive and negative directions $[\mathrm{C} 8=$ 0.0357 (15) $\AA$; $\mathrm{N} 2=-0.0319(14) \AA]$. The $\mathrm{O} 1=\mathrm{C} 8$ bond length of the pyridazinone carbonyl function is 1.230 (3) $\AA$, and the $\mathrm{N} 1-\mathrm{N} 2$ bond length in the pyridazine ring is 1.362 (2) $\AA$, both in accordance with values for related pyridazinones.

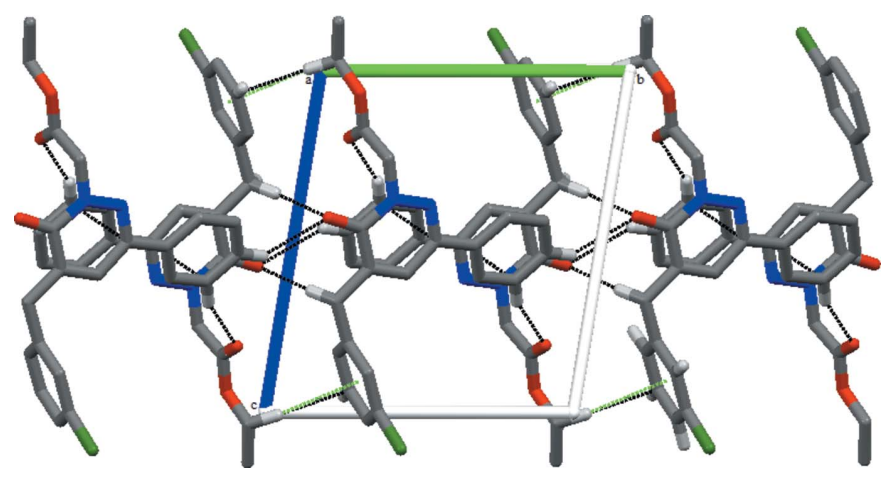

Figure 2

A view along the $a$ axis of the crystal structure of the title compound. Black dashed lines symbolize intermolecular $\mathrm{C}-\mathrm{H} \cdots \mathrm{O}$ hydrogen bonds; $\mathrm{C}-\mathrm{H} \cdots \pi$ interactions are shown as green dashes lines.
Table 1

Hydrogen-bond geometry $\left(\AA,^{\circ}\right)$.

$C g 2$ is the centroid of the C1-C6 phenyl ring

\begin{tabular}{lllll}
\hline$D-\mathrm{H} \cdots A$ & $D-\mathrm{H}$ & $\mathrm{H} \cdots A$ & $D \cdots A$ & $D-\mathrm{H} \cdots A$ \\
\hline $\mathrm{C} 14-\mathrm{H} 14 \cdots \mathrm{O} 2^{\mathrm{i}}$ & 0.93 & 2.53 & $3.416(3)$ & 160 \\
$\mathrm{C} 7-\mathrm{H} 7 B \cdots \mathrm{O} 1^{\text {ii }}$ & 0.97 & 2.54 & $3.485(3)$ & 164 \\
$\mathrm{C} 15-\mathrm{H} 15 \cdots \mathrm{O} 1^{\mathrm{iii}}$ & 0.93 & 2.66 & $3.474(3)$ & 147 \\
$\mathrm{C} 20-\mathrm{H} 20 B \cdots C g 2^{\mathrm{iv}}$ & 0.97 & 2.81 & $3.759(3)$ & 165 \\
\hline
\end{tabular}

Symmetry codes: (i) $-x,-y+1,-z+1$; (ii) $\quad-x+1,-y+2,-z+1$; $\quad$ (iii) $x-1, y-1, z ;$ (iv) $-x+1,-y,-z+1$.

\section{Supramolecular features}

The crystal packing exhibits $\mathrm{C}-\mathrm{H} \cdots \mathrm{O}$ hydrogen bonds between aryl or methylene groups and carbonyl $\mathrm{O}$ atoms (Table 1 ), as well as $\mathrm{C}-\mathrm{H} \cdots \pi$ interactions and van der Waals contacts. Intermolecular $\mathrm{C} 7-\mathrm{H} 7 \mathrm{~B} \cdots \mathrm{O} 1$ and $\mathrm{C} 14-\mathrm{H} 14 \cdots \mathrm{O} 2$ hydrogen bonds produce $R_{2}^{2}(10)$ and $R_{2}^{2}(24)$ motif rings (Fig. 2), supplemented by $\mathrm{C} 15-\mathrm{H} 15 \cdots \mathrm{O} 1$ contacts, forming chains extending parallel to the $c$ axis (Fig. 2). A weak $\mathrm{C} 20-$ $\mathrm{H} 20 B \cdots C g 2(-x+1,-y,-z+1 ; C g 2$ is the centroid of the C1-C6 phenyl ring) contact is also present in this chain (Table 1; Fig. 2). Weak aromatic $\pi-\pi$ stacking interactions between adjacent pyridazine rings $[C g 1 \cdots C g 1(-x+1,-y+1$, $-z+1)=3.8833(13) \AA$, where $C g 1$ is the centroid of the C8C11/N1/N2 ring] along the $a$ axis lead to the formation of a three-dimensional network.

\section{Database survey}

A search of the Cambridge Structural Database (CSD, version 5.40, update November 2018; Groom et al., 2016) revealed two structures containing a similar pyridazinone moiety as in the title structure but with different substituents, viz. 4-benzyl-6- $p$ tolylpyridazin-3(2H)-one (YOTVIN; Oubair et al., 2009) and ethyl 3-methyl-6-oxo-5-(3-(trifluoromethyl)phenyl)-1,6-dihydro-1-pyridazineacetate (QANVOR; Xu et al., 2005). In the crystal structure of YOTVIN, the molecules are connected two-by-two through $\mathrm{N}-\mathrm{H} \cdots \mathrm{O}$ hydrogen bonds with an $R_{2}^{2}(8)$ graph-set motif, forming dimers arranged around an inversion center. Weak $\mathrm{C}-\mathrm{H} \cdots \mathrm{O}$ hydrogen bonds and weak offset $\pi-\pi$ stacking stabilize the packing. In the crystal structure of QANVOR, the phenyl and pyridazinone rings are approximately co-planar, making a dihedral angle of $4.84(13)^{\circ}$. Centrosymmetrically related molecules form dimers through non-classical intermolecular $\mathrm{C}-\mathrm{H}$.. O hydrogen bonds.

\section{Hirshfeld surface analysis}

A Hirshfeld surface analysis (Spackman \& Jayatilaka, 2009) and the associated two-dimensional fingerprint plots (McKinnon et al., 2007) were performed with CrystalExplorer17 (Turner et al., 2017), using a standard (high) surface resolution with the three-dimensional $d_{\text {norm }}$ surfaces plotted over a fixed colour scale of -0.1647 (red) to 1.1730 (blue) a.u. The three-dimensional $d_{\text {norm }}$ surface of the title 
molecule is illustrated in Fig. 3a. The pale-red spots symbolize short contacts and negative $d_{\text {norm }}$ values on the surface and correspond to the $\mathrm{C}-\mathrm{H} \cdots \mathrm{O}$ interactions (Table 1).

The shape-index map of the title molecule was generated in the range -1 to $1 \AA$ (Fig. $3 b$ ). The convex blue regions symbolize hydrogen-donor groups and the concave red regions hydrogen-acceptor groups. $\pi-\pi$ interactions are generally indicated by adjacent red and blue triangles in the shape-index map, as is the case for the title molecule.

The curvedness map of the title complex was generated in the range -4.0 to $0.4 \AA$ (Fig. $3 c$ ). The curvedness plot of the title complex shows large regions of green with a relatively flat (i.e. planar) surface area, indicating the presence of $\pi-\pi$ stacking interactions, while the blue regions demonstrate areas of curvature.

The overall two-dimensional fingerprint plot is illustrated in Fig. $4 a$, delineated into $\mathrm{H} \cdots \mathrm{H}, \mathrm{H} \cdots \mathrm{C} / \mathrm{C} \cdots \mathrm{H}, \mathrm{H} \cdots \mathrm{O} / \mathrm{O} \cdots \mathrm{H}$, $\mathrm{H} \cdots \mathrm{Cl} / \mathrm{Cl} \cdots \mathrm{H}, \mathrm{C} \cdots \mathrm{C}$ contacts associated with their relative contributions to the Hirshfeld surface in Fig. $4 b-f$, respectively. The most important intermolecular interaction is $\mathrm{H} \cdots \mathrm{H}$, contributing $44.5 \%$ to the overall crystal packing, with the centre of the peak $d_{\mathrm{e}}=d_{\mathrm{i}}=1.18 \AA$ (Fig. $4 b$ ). $\mathrm{H} \cdots \mathrm{C} / \mathrm{C} \cdots \mathrm{H}$ contacts, with a $18.5 \%$ contribution to the Hirshfeld surface, indicate the presence of the weak $\mathrm{C}-\mathrm{H} \cdots \pi$ interaction (Table 1). Two pairs of characteristic wings in the fingerprint plot with pairs of tips at $d_{\mathrm{e}}+d_{\mathrm{i}} \sim 2.8 \AA$ are present (Fig. $4 c$ ). $\mathrm{H} \cdots \mathrm{O} / \mathrm{O} \cdots \mathrm{H}$ contacts arising from intermolecular $\mathrm{C}-\mathrm{H} \cdots \mathrm{O}$ hydrogen bonding make a $15.6 \%$ contribution to the Hirshfeld surface and are represented by a pair of sharp spikes in the region $d_{\mathrm{e}}+d_{\mathrm{i}} \sim 2.35 \AA$ The C $\cdots$ C contacts are a measure of $\pi-$ Ip stacking interactions and contribute $2.8 \%$ of the Hirshfeld surface. They appear as an arrow-shaped distribution at $d_{\mathrm{e}}+d_{\mathrm{i}}$ $\sim 3.3 \AA$. Another contact to the Hirshfeld surface is from $\mathrm{H} \cdots \mathrm{Cl} / \mathrm{Cl} \cdots \mathrm{H}$ interactions (10.6\%).

\section{Synthesis and crystallization}

To a solution $(0.99 \mathrm{~g}, 3 \mathrm{mmol})$ of 4-(3-dichlorobenzyl)-6phenylpyridazin-3 $(2 H)$-one in $30 \mathrm{ml}$ of tetrahydrofuran (THF), potassium carbonate $(0.5 \mathrm{~g}, 3.5 \mathrm{mmol})$ was added. The mixture was refluxed for $1 \mathrm{~h}$. After cooling, ethyl bromoacetate $(0.66 \mathrm{~g}, 4 \mathrm{mmol})$ was added and the mixture was refluxed for $8 \mathrm{~h}$. The precipitated material was removed by

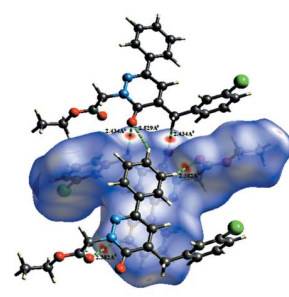

(a)

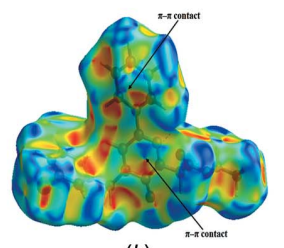

(b)

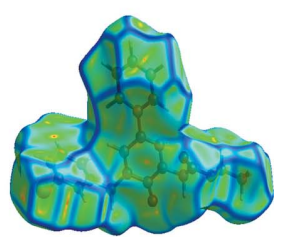

(c)
Figure 3

(a) $d_{\text {norm }}$ mapped on the Hirshfeld surface for visualizing the intermolecular interactions, $(b)$ shape-index map of the title compound and (c) curvedness map of the title compound.

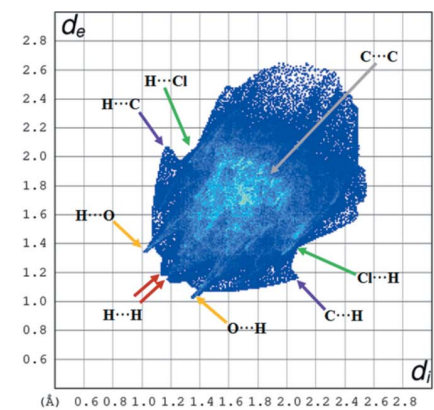

(a)

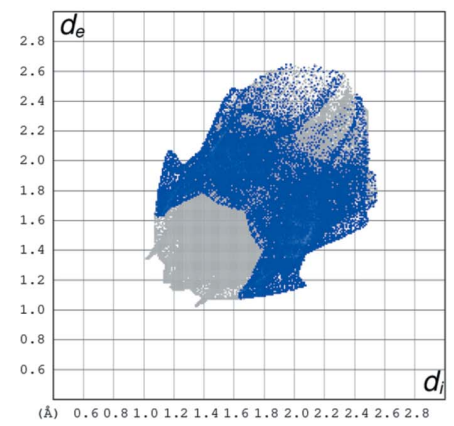

(c)

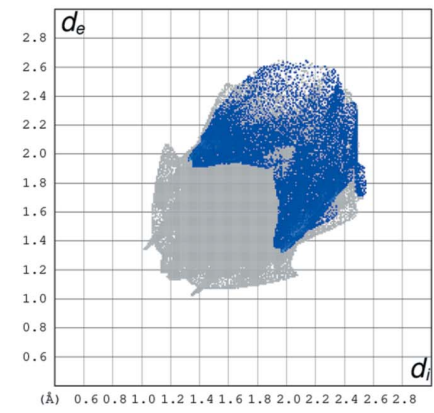

(e)

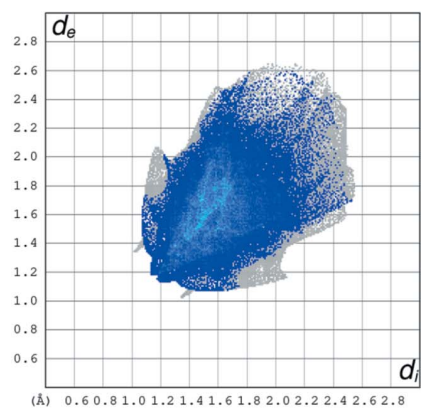

(b)

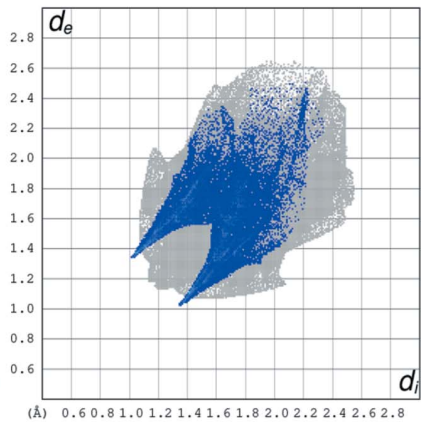

(d)

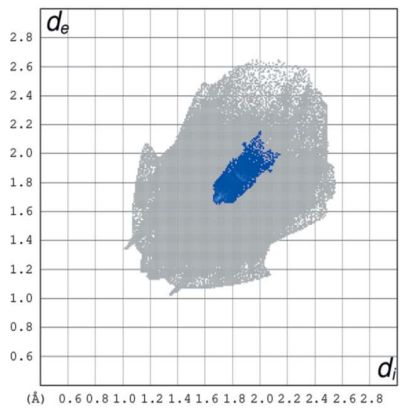

(f)
Figure 4

(a) The overall two-dimensional fingerprint plot, and delineated into $(b)$ $\mathrm{H} \cdots \mathrm{H},(c) \mathrm{H} \cdots \mathrm{C} / \mathrm{C} \cdots \mathrm{H},(d) \mathrm{H} \cdots \mathrm{O} / \mathrm{O} \cdots \mathrm{H},(e) \mathrm{H} \cdots \mathrm{Cl} / \mathrm{Cl} \cdots \mathrm{H}$ and $(f)$ C. C interactions.

filtration and the solvent evaporated under vacuum. The residue was purified through silica gel column chromatography using hexane/ethyl acetate $(4: 6 v / v)$. Slow evaporation at room temperature led to formation of single crystals with a yield of $70 \%$.

\section{Refinement}

Crystal data, data collection and structure refinement details are summarized in Table 2. Hydrogen atoms were fixed geometrically and treated as riding, with $\mathrm{C}-\mathrm{H}=0.97 \AA$ for methyl $\left[U_{\text {iso }}(\mathrm{H})=1.2 U_{\text {eq }}(\mathrm{C})\right], \mathrm{C}-\mathrm{H}=0.96 \AA$ for methylene $\left[U_{\text {iso }}(\mathrm{H})=1.5 U_{\text {eq }}(\mathrm{C})\right], \mathrm{C}-\mathrm{H}=0.93 \AA$ for aromatic $\left[U_{\text {iso }}(\mathrm{H})=\right.$ $\left.1.2 U_{\mathrm{eq}}(\mathrm{C})\right]$ and $\mathrm{C}-\mathrm{H}=0.98 \AA$ for methine $\left[U_{\text {iso }}(\mathrm{H})=\right.$ $\left.1.2 U_{\text {eq }}(\mathrm{C})\right] \mathrm{H}$ atoms. 
Table 2

Experimental details.

\begin{tabular}{|c|c|}
\hline \multicolumn{2}{|l|}{ Crystal data } \\
\hline Chemical formula & $\mathrm{C}_{21} \mathrm{H}_{19} \mathrm{ClN}_{2} \mathrm{O}_{3}$ \\
\hline$M_{\mathrm{r}}$ & 382.83 \\
\hline Crystal system, space group & Triclinic, $P \overline{1}$ \\
\hline Temperature $(\mathrm{K})$ & 296 \\
\hline$a, b, c(\AA)$ & $\begin{array}{l}8.8410(11), 10.3043(12) \\
\quad 11.3610(12)\end{array}$ \\
\hline$\alpha, \beta, \gamma\left({ }^{\circ}\right)$ & $94.801(9), 103.596(9), 106.905(9)$ \\
\hline$V\left(\AA^{3}\right)$ & $949.6(2)$ \\
\hline$Z$ & 2 \\
\hline Radiation type & Мо $K \alpha$ \\
\hline$\mu\left(\mathrm{mm}^{-1}\right)$ & 0.23 \\
\hline Crystal size $(\mathrm{mm})$ & $0.88 \times 0.53 \times 0.19$ \\
\hline \multicolumn{2}{|l|}{ Data collection } \\
\hline Diffractometer & Stoe IPDS 2 \\
\hline Absorption correction & $\begin{array}{l}\text { Integration ( } X \text {-RED32; Stoe \& Cie, } \\
\text { 2002) }\end{array}$ \\
\hline$T_{\min }, T_{\max }$ & $0.876,0.960$ \\
\hline $\begin{array}{l}\text { No. of measured, independent and } \\
\text { observed }[I>2 \sigma(I)] \text { reflections }\end{array}$ & $9612,3716,2058$ \\
\hline$R_{\text {int }}$ & 0.031 \\
\hline$(\sin \theta / \lambda)_{\max }\left(\AA^{-1}\right)$ & 0.617 \\
\hline \multicolumn{2}{|l|}{ Refinement } \\
\hline$R\left[F^{2}>2 \sigma\left(F^{2}\right)\right], w R\left(F^{2}\right), S$ & $0.047,0.127,0.91$ \\
\hline No. of reflections & 3716 \\
\hline No. of parameters & 245 \\
\hline $\mathrm{H}$-atom treatment & H-atom parameters constrained \\
\hline$\Delta \rho_{\max }, \Delta \rho_{\min }\left(\mathrm{e} \AA^{-3}\right)$ & $0.26,-0.34$ \\
\hline
\end{tabular}

Computer programs: X-AREA and X-RED (Stoe \& Cie, 2002), SHELXT2017 (Sheldrick, 2015a), SHELXL2018 (Sheldrick, 2015b), Mercury (Macrae et al., 2008), WinGX (Farrugia, 2012), PLATON (Spek, 2009) and publCIF (Westrip, 2010).

\section{Acknowledgements}

The authors acknowledge the Faculty of Arts and Sciences, Ondokuz Mayıs University, Turkey, for the use of the Stoe IPDS 2 diffractometer (purchased under grant F.279 of the University Research Fund).

\section{References}

Akhtar, W., Shaquiquzzaman, M., Akhter, M., Verma, G., Khan, M. F. \& Alam, M. M. (2016). Eur. J. Med. Chem. 123, 256-281.

Asif, M. (2013). Mini-Rev. Org. Chem. 10, 113-122.

Asif, M. (2015). Mini Rev. Med. Chem. 14, 1093-1103.

Barberot, C., Moniot, A., Allart-Simon, I., Malleret, L., Yegorova, T., Laronze-Cochard, M., Bentaher, A., Médebielle, M., Bouillon, J. P., Hénon, E., Sapi, J., Velard, F. \& Gérard, S. (2018). Eur. J. Med. Chem. 146, 139-146.

Boukharsa, Y., Meddah, B., Tiendrebeogo, R. Y., Ibrahimi, A., Taoufik, J., Cherrah, Y., Benomar, A., Faouzi, M. E. A. \& Ansar, M. (2016). Med. Chem. Res. 25, 494-500.

Chkirate, K., Kansiz, S., Karrouchi, K., Mague, J. T., Dege, N. \& Essassi, E. M. (2019a). Acta Cryst. E75, 154-158.

Chkirate, K., Kansiz, S., Karrouchi, K., Mague, J. T., Dege, N. \& Essassi, E. M. (2019b). Acta Cryst. E75, 33-37.
Dubey, S. \& Bhosle, P. A. (2015). Med. Chem. Res. 24, 3579-3598.

Farrugia, L. J. (2012). J. Appl. Cryst. 45, 849-854.

Gökçe, M., Utku, S. \& Küpeli, E. (2009). Eur. J. Med. Chem. 44, 37603764.

Groom, C. R., Bruno, I. J., Lightfoot, M. P. \& Ward, S. C. (2016). Acta Cryst. B72, 171-179.

Karrouchi, K., Ansar, M., Radi, S., Saadi, M. \& El Ammari, L. (2015). Acta Cryst. E71, o890-0891.

Karrouchi, K., Radi, S., Ansar, M. H., Taoufik, J., Ghabbour, H. A. \& Mabkhot, Y. N. (2016a). Z. Kristallogr. New Cryst. Struct. 231, $883-$ 886.

Karrouchi, K., Radi, S., Ansar, M. H., Taoufik, J., Ghabbour, H. A. \& Mabkhot, Y. N. (2016b). Z. Kristallogr. New Cryst. Struct. 231, 839841.

Livermore, D., Bethell, R. C., Cammack, N., Hancock, A. P., Hann, M. M., Green, D., Lamont, R. B., Noble, S. A., Orr, D. C. \& Payne, J. J. (1993). J. Med. Chem. 36, 3784-3794.

Macrae, C. F., Bruno, I. J., Chisholm, J. A., Edgington, P. R., McCabe, P., Pidcock, E., Rodriguez-Monge, L., Taylor, R., van de Streek, J. \& Wood, P. A. (2008). J. Appl. Cryst. 41, 466-470.

McKinnon, J. J., Jayatilaka, D. \& Spackman, M. A. (2007). Chem. Commun. pp. 3814-3816.

Ochiai, K., Takita, S., Eiraku, T., Kojima, A., Iwase, K., Kishi, T., Fukuchi, K., Yasue, T., Adams, D. R., Allcock, R. W., Jiang, Z. \& Kohno, Y. (2012). Bioorg. Med. Chem. 20, 1644-1658.

Oubair, A., Daran, J.-C., Fihi, R., Majidi, L. \& Azrour, M. (2009). Acta Cryst. E65, o1350-o1351.

Partap, S., Akhtar, M. J., Yar, M. S., Hassan, M. Z. \& Siddiqui, A. A. (2018). Bioorg. Chem. 77, 74-83.

Sharma, B., Verma, A., Sharma, U. K. \& Prajapati, S. (2014). Med. Chem. Res. 23, 146-157.

Sheldrick, G. M. (2015a). Acta Cryst. A71, 3-8.

Sheldrick, G. M. (2015b). Acta Cryst. C71, 3-8.

Siddiqui, A. A., Mishra, R., Shaharyar, M., Husain, A., Rashid, M. \& Pal, P. (2011). Bioorg. Med. Chem. Lett. 21, 1023-1026.

Sönmez, M., Berber, I. \& Akbaş, E. (2006). Eur. J. Med. Chem. 41, 101-105.

Spackman, M. A. \& Jayatilaka, D. (2009). CrystEngComm, 11, 19-32. Spek, A. L. (2009). Acta Cryst. D65, 148-155.

Stoe \& Cie (2002). $X$-AREA and $X$-RED32. Stoe \& Cie GmbH, Darmstadt, Germany.

Tao, M., Aimone, L. D., Gruner, J. A., Mathiasen, J. R., Huang, Z., Lyons, J., Raddatz, R. \& Hudkins, R. L. (2012). Bioorg. Med. Chem. Lett. 22, 1073-1077.

Thakur, A. S., Verma, P. \& Chandy, A. (2010). Asian. J. Res. Chem, 3, 265-271.

Turner, M. J., McKinnon, J. J., Wolff, S. K., Grimwood, D. J., Spackman, P. R., Jayatilaka, D. \& Spackman, M. A. (2017). CrystalExplorer17. University of Western Australia. http://hirshfeldsurface.net.

Wang, T., Dong, Y., Wang, L.-C., Xiang, B.-R., Chen, Z. \& Qu, L.-B. (2008). Arzneimittelforschung, 58, 569-573.

Westrip, S. P. (2010). J. Appl. Cryst. 43, 920-925.

Xu, H., Song, H.-B., Yao, C.-S., Zhu, Y.-Q., Hu, F.-Z., Zou, X.-M. \& Yang, H.-Z. (2005). Acta Cryst. E61, o1561-o1563.

Zhou, G., Ting, P. C., Aslanian, R., Cao, J., Kim, D. W., Kuang, R., Lee, J. F., Schwerdt, J., Wu, H., Jason Herr, R., Zych, A. J., Yang, J., Lam, S., Wainhaus, S., Black, T. A., McNicholas, P. M., Xu, Y. \& Walker, S. S. (2011). Bioorg. Med. Chem. Lett. 21, 2890-2893. 


\section{supporting information}

Acta Cryst. (2019). E75, 892-895 [https://doi.org/10.1107/S2056989019007424]

Crystal structure and Hirshfeld surface analysis of ethyl 2-[5-(3-chlorobenzyl)-6oxo-3-phenyl-1,6-dihydropyridazin-1-yl]acetate

Fouad El Kalai, Cemile Baydere, Said Daoui, Rafik Saddik, Necmi Dege, Khalid Karrouchi and Noureddine Benchat

Computing details

Data collection: $X$-AREA (Stoe \& Cie, 2002); cell refinement: $X$-AREA (Stoe \& Cie, 2002); data reduction: $X$-RED (Stoe \& Cie, 2002); program(s) used to solve structure: SHELXT2017 (Sheldrick, 2015a); program(s) used to refine structure: SHELXL2018 (Sheldrick, 2015b); molecular graphics: Mercury (Macrae et al., 2008) and PLATON (Spek, 2009); software used to prepare material for publication: WinGX (Farrugia, 2012), SHELXL2018 (Sheldrick, 2015b), PLATON (Spek, 2009) and publCIF (Westrip, 2010).

Ethyl 2-[5-(3-chlorobenzyl)-6-oxo-3-phenyl-1,6-dihydropyridazin-1-yl]acetate

Crystal data

$\mathrm{C}_{21} \mathrm{H}_{19} \mathrm{ClN}_{2} \mathrm{O}_{3}$

$M_{r}=382.83$

Triclinic, $P \overline{1}$

$a=8.8410(11) \AA$

$b=10.3043(12) \AA$

$c=11.3610(12) \AA$

$\alpha=94.801(9)^{\circ}$

$\beta=103.596(9)^{\circ}$

$\gamma=106.905(9)^{\circ}$

$V=949.6(2) \AA^{3}$

Data collection

Stoe IPDS 2

diffractometer

Detector resolution: 6.67 pixels $\mathrm{mm}^{-1}$

rotation method scans

Absorption correction: integration

(X-RED32; Stoe \& Cie, 2002)

$T_{\text {min }}=0.876, T_{\text {max }}=0.960$

9612 measured reflections

\section{Refinement}

Refinement on $F^{2}$

Least-squares matrix: full

$R\left[F^{2}>2 \sigma\left(F^{2}\right)\right]=0.047$

$w R\left(F^{2}\right)=0.127$

$S=0.91$

3716 reflections
$Z=2$

$F(000)=400$

$D_{\mathrm{x}}=1.339 \mathrm{Mg} \mathrm{m}^{-3}$

Mo $K \alpha$ radiation, $\lambda=0.71073 \AA$

Cell parameters from 11025 reflections

$\theta=3.0-31.4^{\circ}$

$\mu=0.23 \mathrm{~mm}^{-1}$

$T=296 \mathrm{~K}$

Prism, yellow

$0.88 \times 0.53 \times 0.19 \mathrm{~mm}$

3716 independent reflections

2058 reflections with $I>2 \sigma(I)$

$R_{\text {int }}=0.031$

$\theta_{\text {max }}=26.0^{\circ}, \theta_{\min }=3.0^{\circ}$

$h=-10 \rightarrow 10$

$k=-12 \rightarrow 12$

$l=-14 \rightarrow 14$

245 parameters

0 restraints

Hydrogen site location: inferred from neighbouring sites

$\mathrm{H}$-atom parameters constrained 
$w=1 /\left[\sigma^{2}\left(F_{\mathrm{o}}^{2}\right)+(0.0666 P)^{2}\right]$

where $P=\left(F_{\mathrm{o}}^{2}+2 F_{\mathrm{c}}^{2}\right) / 3$

$(\Delta / \sigma)_{\max }<0.001$
$\Delta \rho_{\max }=0.26 \mathrm{e} \AA^{-3}$
$\Delta \rho_{\min }=-0.34 \mathrm{e} \AA^{-3}$

Special details

Geometry. All esds (except the esd in the dihedral angle between two 1.s. planes) are estimated using the full covariance matrix. The cell esds are taken into account individually in the estimation of esds in distances, angles and torsion angles; correlations between esds in cell parameters are only used when they are defined by crystal symmetry. An approximate (isotropic) treatment of cell esds is used for estimating esds involving l.s. planes.

Fractional atomic coordinates and isotropic or equivalent isotropic displacement parameters $\left(\hat{A}^{2}\right)$

\begin{tabular}{|c|c|c|c|c|}
\hline & $x$ & $y$ & $z$ & $U_{\text {iso }} * / U_{\text {eq }}$ \\
\hline $\mathrm{C} 11$ & $0.29831(14)$ & $0.53925(11)$ & $-0.10674(8)$ & $0.1264(4)$ \\
\hline $\mathrm{O} 3$ & 0.72303 (19) & $0.86361(16)$ & $0.93869(13)$ & $0.0654(5)$ \\
\hline $\mathrm{O} 2$ & $0.4976(2)$ & $0.87323(18)$ & $0.80559(15)$ & $0.0704(5)$ \\
\hline $\mathrm{O} 1$ & $0.6649(2)$ & 0.89797 (17) & $0.57180(15)$ & $0.0725(5)$ \\
\hline N1 & $0.4130(2)$ & $0.57611(18)$ & $0.60996(15)$ & $0.0503(5)$ \\
\hline N2 & $0.5368(2)$ & $0.69655(18)$ & $0.62523(15)$ & $0.0542(5)$ \\
\hline $\mathrm{C} 12$ & $0.1658(3)$ & 0.4089 (2) & $0.49033(17)$ & $0.0463(5)$ \\
\hline $\mathrm{C} 11$ & $0.3033(3)$ & $0.5381(2)$ & $0.50375(17)$ & $0.0462(5)$ \\
\hline C19 & $0.6111(3)$ & $0.8315(2)$ & $0.83046(19)$ & $0.0536(6)$ \\
\hline $\mathrm{C} 10$ & $0.3151(3)$ & $0.6166(2)$ & $0.40745(18)$ & $0.0507(5)$ \\
\hline $\mathrm{H} 10$ & 0.238158 & 0.583782 & 0.331377 & $0.061 *$ \\
\hline C9 & $0.4352(3)$ & $0.7377(2)$ & $0.42376(19)$ & $0.0528(6)$ \\
\hline C13 & 0.0216 & $0.3784(2)$ & 0.39727 (19) & $0.0556(6)$ \\
\hline H13 & 0.012681 & 0.437926 & 0.340794 & $0.067 *$ \\
\hline $\mathrm{C} 8$ & $0.5539(3)$ & $0.7869(2)$ & $0.5427(2)$ & $0.0557(6)$ \\
\hline $\mathrm{C} 5$ & $0.3334(3)$ & $0.7688(2)$ & $0.2046(2)$ & $0.0602(6)$ \\
\hline $\mathrm{C} 17$ & $0.1752(3)$ & $0.3177(2)$ & $0.5723(2)$ & $0.0617(6)$ \\
\hline $\mathrm{H} 17$ & 0.270130 & 0.335520 & 0.636054 & $0.074 *$ \\
\hline $\mathrm{C} 18$ & $0.6531(3)$ & $0.7357(2)$ & $0.7459(2)$ & $0.0618(6)$ \\
\hline H18A & 0.762208 & 0.780076 & 0.737838 & $0.074 *$ \\
\hline H18B & 0.654649 & 0.653680 & 0.781667 & $0.074 *$ \\
\hline C14 & -0.1095 & $0.2608(3)$ & $0.3867(2)$ & $0.0659(7)$ \\
\hline H14 & -0.206077 & 0.242734 & 0.324506 & $0.079 *$ \\
\hline C6 & $0.3669(3)$ & $0.6883(3)$ & $0.1175(2)$ & $0.0693(7)$ \\
\hline H6 & 0.465811 & 0.669538 & 0.134709 & $0.083^{*}$ \\
\hline $\mathrm{C} 15$ & $-0.0967(4)$ & 0.1717 (3) & $0.4678(3)$ & $0.0735(7)$ \\
\hline H15 & -0.183752 & 0.091911 & 0.460255 & $0.088^{*}$ \\
\hline $\mathrm{C} 7$ & 0.4550 & $0.8283(2)$ & $0.3280(2)$ & $0.0659(7)$ \\
\hline H7A & 0.565102 & 0.846300 & 0.318471 & $0.079 *$ \\
\hline H7B & 0.444144 & 0.915570 & 0.356666 & $0.079^{*}$ \\
\hline $\mathrm{C} 20$ & $0.6983(3)$ & $0.9534(3)$ & $1.0330(2)$ & $0.0697(7)$ \\
\hline $\mathrm{H} 20 \mathrm{~A}$ & 0.592464 & 0.912468 & 1.048073 & $0.084 *$ \\
\hline $\mathrm{H} 20 \mathrm{~B}$ & 0.702185 & 1.041629 & 1.007747 & $0.084^{*}$ \\
\hline C16 & $0.0439(4)$ & $0.1999(3)$ & $0.5596(3)$ & $0.0749(8)$ \\
\hline H16 & 0.051884 & 0.138934 & 0.614912 & $0.090 *$ \\
\hline $\mathrm{C} 1$ & 0.2528 & $0.6351(3)$ & $0.0037(2)$ & $0.0752(8)$ \\
\hline
\end{tabular}




$\begin{array}{lllll}\mathrm{C} 4 & 0.1844(4) & 0.7928(3) & 0.1767(2) & 0.0752(8) \\ \mathrm{H} 4 & 0.160060 & 0.847345 & 0.234301 & 0.090^{*} \\ \mathrm{C} 2 & 0.1061(4) & 0.6593(3) & -0.0218(3) & 0.0839(9) \\ \mathrm{H} 2 & 0.030067 & 0.622809 & -0.097796 & 0.101^{*} \\ \mathrm{C} 3 & 0.0711(4) & 0.7371(3) & 0.0646(3) & 0.0902(9) \\ \mathrm{H} 3 & -0.029726 & 0.752677 & 0.047719 & 0.108^{*} \\ \mathrm{C} 21 & 0.8330(5) & 0.9709(4) & 1.1455(3) & 0.1279(16) \\ \mathrm{H} 21 \mathrm{~A} & 0.936252 & 1.019422 & 1.131745 & 0.192^{*} \\ \mathrm{H} 21 \mathrm{~B} & 0.834403 & 0.882260 & 1.164831 & 0.192^{*} \\ \mathrm{H} 21 \mathrm{C} & 0.815587 & 1.022374 & 1.212596 & 0.192^{*}\end{array}$

Atomic displacement parameters $\left(\AA^{2}\right)$

\begin{tabular}{lllllll}
\hline & $U^{11}$ & $U^{22}$ & $U^{33}$ & $U^{12}$ & $U^{13}$ & $U^{23}$ \\
\hline C11 & $0.1545(9)$ & $0.1266(8)$ & $0.0925(6)$ & $0.0370(7)$ & $0.0468(6)$ & $-0.0221(5)$ \\
O3 & $0.0619(10)$ & $0.0768(11)$ & $0.0468(8)$ & $0.0251(9)$ & $-0.0023(8)$ & $-0.0084(7)$ \\
O2 & $0.0597(11)$ & $0.0818(12)$ & $0.0620(10)$ & $0.0286(9)$ & $0.0007(8)$ & $-0.0056(8)$ \\
O1 & $0.0687(11)$ & $0.0536(10)$ & $0.0731(11)$ & $-0.0058(9)$ & $0.0156(9)$ & $-0.0070(8)$ \\
N1 & $0.0517(11)$ & $0.0514(11)$ & $0.0437(10)$ & $0.0153(9)$ & $0.0090(8)$ & $0.0008(8)$ \\
N2 & $0.0504(11)$ & $0.0545(11)$ & $0.0460(10)$ & $0.0091(10)$ & $0.0048(8)$ & $-0.0036(8)$ \\
C12 & $0.0519(13)$ & $0.0456(12)$ & $0.0411(11)$ & $0.0144(10)$ & $0.0158(10)$ & $0.0004(9)$ \\
C11 & $0.0502(13)$ & $0.0478(12)$ & $0.0386(11)$ & $0.0162(11)$ & $0.0100(10)$ & $0.0005(9)$ \\
C19 & $0.0475(13)$ & $0.0558(13)$ & $0.0452(12)$ & $0.0093(11)$ & $0.0012(10)$ & $0.0008(9)$ \\
C10 & $0.0563(14)$ & $0.0501(13)$ & $0.0401(11)$ & $0.0141(11)$ & $0.0086(10)$ & $0.0009(9)$ \\
C9 & $0.0594(14)$ & $0.0458(12)$ & $0.0505(12)$ & $0.0126(11)$ & $0.0175(11)$ & $0.0015(9)$ \\
C13 & $0.0589(14)$ & $0.0537(13)$ & $0.0475(12)$ & $0.0142(12)$ & $0.0098(11)$ & $-0.0004(10)$ \\
C8 & $0.0573(15)$ & $0.0511(13)$ & $0.0525(13)$ & $0.0119(12)$ & $0.0145(11)$ & $-0.0035(10)$ \\
C5 & $0.0752(17)$ & $0.0482(13)$ & $0.0541(13)$ & $0.0100(12)$ & $0.0218(12)$ & $0.0158(10)$ \\
C17 & $0.0624(16)$ & $0.0636(15)$ & $0.0562(13)$ & $0.0174(13)$ & $0.0130(12)$ & $0.0126(11)$ \\
C18 & $0.0541(14)$ & $0.0672(15)$ & $0.0505(12)$ & $0.0165(12)$ & $-0.0014(11)$ & $-0.0082(11)$ \\
C14 & $0.0514(15)$ & $0.0637(16)$ & $0.0668(15)$ & $0.0067(13)$ & $0.0084(12)$ & $-0.0105(13)$ \\
C6 & $0.0742(18)$ & $0.0634(15)$ & $0.0690(16)$ & $0.0162(13)$ & $0.0246(14)$ & $0.0100(12)$ \\
C15 & $0.0699(18)$ & $0.0566(16)$ & $0.0838(18)$ & $0.0018(13)$ & $0.0295(15)$ & $-0.0022(14)$ \\
C7 & $0.0793(18)$ & $0.0524(14)$ & $0.0592(14)$ & $0.0114(12)$ & $0.0179(13)$ & $0.0110(11)$ \\
C20 & $0.0812(19)$ & $0.0734(17)$ & $0.0524(13)$ & $0.0277(14)$ & $0.0147(13)$ & $-0.0022(12)$ \\
C16 & $0.091(2)$ & $0.0592(16)$ & $0.0750(17)$ & $0.0149(16)$ & $0.0318(16)$ & $0.0214(13)$ \\
C1 & $0.095(2)$ & $0.0646(16)$ & $0.0622(16)$ & $0.0137(16)$ & $0.0299(15)$ & $0.0051(12)$ \\
C4 & $0.089(2)$ & $0.0779(18)$ & $0.0639(16)$ & $0.0308(16)$ & $0.0244(15)$ & $0.0151(13)$ \\
C2 & $0.092(2)$ & $0.085(2)$ & $0.0601(16)$ & $0.0142(17)$ & $0.0099(16)$ & $0.0108(14)$ \\
C3 & $0.088(2)$ & $0.101(2)$ & $0.083(2)$ & $0.0356(18)$ & $0.0183(17)$ & $0.0193(17)$ \\
C21 & $0.156(3)$ & $0.171(4)$ & $0.0489(16)$ & $0.091(3)$ & $-0.0186(19)$ & $-0.0337(18)$ \\
& & & & & & \\
\hline & & & & & &
\end{tabular}

Geometric parameters $\left(A,{ }^{\circ}\right)$

\begin{tabular}{llll}
\hline $\mathrm{C} 11-\mathrm{C} 1$ & $1.724(3)$ & $\mathrm{C} 17-\mathrm{H} 17$ & 0.9300 \\
$\mathrm{O} 3-\mathrm{C} 19$ & $1.331(2)$ & $\mathrm{C} 18-\mathrm{H} 18 \mathrm{~A}$ & 0.9700 \\
$\mathrm{O} 3-\mathrm{C} 20$ & $1.454(3)$ & $\mathrm{C} 18-\mathrm{H} 18 \mathrm{~B}$ & 0.9700 \\
$\mathrm{O} 2-\mathrm{C} 19$ & $1.187(3)$ & $\mathrm{C} 14-\mathrm{C} 15$ & $1.362(4)$
\end{tabular}




\begin{tabular}{|c|c|c|c|}
\hline $\mathrm{O} 1-\mathrm{C} 8$ & $1.230(3)$ & $\mathrm{C} 14-\mathrm{H} 14$ & 0.9300 \\
\hline $\mathrm{N} 1-\mathrm{C} 11$ & $1.304(2)$ & $\mathrm{C} 6-\mathrm{C} 1$ & $1.391(4)$ \\
\hline $\mathrm{N} 1-\mathrm{N} 2$ & $1.362(2)$ & $\mathrm{C} 6-\mathrm{H} 6$ & 0.9300 \\
\hline $\mathrm{N} 2-\mathrm{C} 8$ & $1.378(3)$ & $\mathrm{C} 15-\mathrm{C} 16$ & $1.359(4)$ \\
\hline $\mathrm{N} 2-\mathrm{C} 18$ & $1.450(3)$ & $\mathrm{C} 15-\mathrm{H} 15$ & 0.9300 \\
\hline $\mathrm{C} 12-\mathrm{C} 17$ & $1.383(3)$ & $\mathrm{C} 7-\mathrm{H} 7 \mathrm{~A}$ & 0.9700 \\
\hline $\mathrm{C} 12-\mathrm{C} 13$ & $1.385(3)$ & $\mathrm{C} 7-\mathrm{H} 7 \mathrm{~B}$ & 0.9700 \\
\hline $\mathrm{C} 12-\mathrm{C} 11$ & $1.487(3)$ & $\mathrm{C} 20-\mathrm{C} 21$ & $1.484(4)$ \\
\hline $\mathrm{C} 11-\mathrm{C} 10$ & $1.420(3)$ & $\mathrm{C} 20-\mathrm{H} 20 \mathrm{~A}$ & 0.9700 \\
\hline $\mathrm{C} 19-\mathrm{C} 18$ & $1.502(3)$ & $\mathrm{C} 20-\mathrm{H} 20 \mathrm{~B}$ & 0.9700 \\
\hline $\mathrm{C} 10-\mathrm{C} 9$ & $1.347(3)$ & $\mathrm{C} 16-\mathrm{H} 16$ & 0.9300 \\
\hline $\mathrm{C} 10-\mathrm{H} 10$ & 0.9300 & $\mathrm{C} 1-\mathrm{C} 2$ & $1.360(4)$ \\
\hline $\mathrm{C} 9-\mathrm{C} 8$ & $1.447(3)$ & $\mathrm{C} 4-\mathrm{C} 3$ & $1.378(4)$ \\
\hline $\mathrm{C} 9-\mathrm{C} 7$ & $1.500(3)$ & $\mathrm{C} 4-\mathrm{H} 4$ & 0.9300 \\
\hline $\mathrm{C} 13-\mathrm{C} 14$ & $1.386(3)$ & $\mathrm{C} 2-\mathrm{C} 3$ & $1.363(4)$ \\
\hline $\mathrm{C} 13-\mathrm{H} 13$ & 0.9300 & $\mathrm{C} 2-\mathrm{H} 2$ & 0.9300 \\
\hline $\mathrm{C} 5-\mathrm{C} 6$ & $1.378(3)$ & $\mathrm{C} 3-\mathrm{H} 3$ & 0.9300 \\
\hline $\mathrm{C} 5-\mathrm{C} 4$ & $1.380(4)$ & $\mathrm{C} 21-\mathrm{H} 21 \mathrm{~A}$ & 0.9600 \\
\hline $\mathrm{C} 5-\mathrm{C} 7$ & $1.503(3)$ & $\mathrm{C} 21-\mathrm{H} 21 \mathrm{~B}$ & 0.9600 \\
\hline $\mathrm{C} 17-\mathrm{C} 16$ & $1.384(4)$ & $\mathrm{C} 21-\mathrm{H} 21 \mathrm{C}$ & 0.9600 \\
\hline $\mathrm{C} 19-\mathrm{O} 3-\mathrm{C} 20$ & $116.13(18)$ & $\mathrm{C} 13-\mathrm{C} 14-\mathrm{H} 14$ & 120.1 \\
\hline $\mathrm{C} 11-\mathrm{N} 1-\mathrm{N} 2$ & $116.83(18)$ & $\mathrm{C} 5-\mathrm{C} 6-\mathrm{C} 1$ & $120.0(3)$ \\
\hline $\mathrm{N} 1-\mathrm{N} 2-\mathrm{C} 8$ & $126.86(17)$ & $\mathrm{C} 5-\mathrm{C} 6-\mathrm{H} 6$ & 120.0 \\
\hline $\mathrm{N} 1-\mathrm{N} 2-\mathrm{C} 18$ & $114.58(19)$ & $\mathrm{C} 1-\mathrm{C} 6-\mathrm{H} 6$ & 120.0 \\
\hline $\mathrm{C} 8-\mathrm{N} 2-\mathrm{C} 18$ & $118.35(19)$ & $\mathrm{C} 16-\mathrm{C} 15-\mathrm{C} 14$ & $119.7(2)$ \\
\hline $\mathrm{C} 17-\mathrm{C} 12-\mathrm{C} 13$ & $117.8(2)$ & $\mathrm{C} 16-\mathrm{C} 15-\mathrm{H} 15$ & 120.1 \\
\hline $\mathrm{C} 17-\mathrm{C} 12-\mathrm{C} 11$ & $121.31(19)$ & $\mathrm{C} 14-\mathrm{C} 15-\mathrm{H} 15$ & 120.1 \\
\hline $\mathrm{C} 13-\mathrm{C} 12-\mathrm{C} 11$ & $120.81(19)$ & $\mathrm{C} 9-\mathrm{C} 7-\mathrm{C} 5$ & $114.18(19)$ \\
\hline $\mathrm{N} 1-\mathrm{C} 11-\mathrm{C} 10$ & $121.6(2)$ & $\mathrm{C} 9-\mathrm{C} 7-\mathrm{H} 7 \mathrm{~A}$ & 108.7 \\
\hline $\mathrm{N} 1-\mathrm{C} 11-\mathrm{C} 12$ & $116.04(18)$ & $\mathrm{C} 5-\mathrm{C} 7-\mathrm{H} 7 \mathrm{~A}$ & 108.7 \\
\hline $\mathrm{C} 10-\mathrm{C} 11-\mathrm{C} 12$ & $122.40(17)$ & $\mathrm{C} 9-\mathrm{C} 7-\mathrm{H} 7 \mathrm{~B}$ & 108.7 \\
\hline $\mathrm{O} 2-\mathrm{C} 19-\mathrm{O} 3$ & $125.0(2)$ & $\mathrm{C} 5-\mathrm{C} 7-\mathrm{H} 7 \mathrm{~B}$ & 108.7 \\
\hline $\mathrm{O} 2-\mathrm{C} 19-\mathrm{C} 18$ & $126.11(19)$ & $\mathrm{H} 7 \mathrm{~A}-\mathrm{C} 7-\mathrm{H} 7 \mathrm{~B}$ & 107.6 \\
\hline $\mathrm{O} 3-\mathrm{C} 19-\mathrm{C} 18$ & $108.9(2)$ & $\mathrm{O} 3-\mathrm{C} 20-\mathrm{C} 21$ & $106.6(2)$ \\
\hline $\mathrm{C} 9-\mathrm{C} 10-\mathrm{C} 11$ & $121.50(19)$ & $\mathrm{O} 3-\mathrm{C} 20-\mathrm{H} 20 \mathrm{~A}$ & 110.4 \\
\hline $\mathrm{C} 9-\mathrm{C} 10-\mathrm{H} 10$ & 119.3 & $\mathrm{C} 21-\mathrm{C} 20-\mathrm{H} 20 \mathrm{~A}$ & 110.4 \\
\hline $\mathrm{C} 11-\mathrm{C} 10-\mathrm{H} 10$ & 119.3 & $\mathrm{O} 3-\mathrm{C} 20-\mathrm{H} 20 \mathrm{~B}$ & 110.4 \\
\hline $\mathrm{C} 10-\mathrm{C} 9-\mathrm{C} 8$ & $118.4(2)$ & $\mathrm{C} 21-\mathrm{C} 20-\mathrm{H} 20 \mathrm{~B}$ & 110.4 \\
\hline $\mathrm{C} 10-\mathrm{C} 9-\mathrm{C} 7$ & $125.0(2)$ & $\mathrm{H} 20 \mathrm{~A}-\mathrm{C} 20-\mathrm{H} 20 \mathrm{~B}$ & 108.6 \\
\hline $\mathrm{C} 8-\mathrm{C} 9-\mathrm{C} 7$ & $116.5(2)$ & $\mathrm{C} 15-\mathrm{C} 16-\mathrm{C} 17$ & $121.1(3)$ \\
\hline $\mathrm{C} 12-\mathrm{C} 13-\mathrm{C} 14$ & $121.3(2)$ & $\mathrm{C} 15-\mathrm{C} 16-\mathrm{H} 16$ & 119.4 \\
\hline $\mathrm{C} 12-\mathrm{C} 13-\mathrm{H} 13$ & 119.4 & $\mathrm{C} 17-\mathrm{C} 16-\mathrm{H} 16$ & 119.4 \\
\hline $\mathrm{C} 14-\mathrm{C} 13-\mathrm{H} 13$ & 119.4 & $\mathrm{C} 2-\mathrm{C} 1-\mathrm{C} 6$ & $120.6(3)$ \\
\hline $\mathrm{O} 1-\mathrm{C} 8-\mathrm{N} 2$ & $120.3(2)$ & $\mathrm{C} 2-\mathrm{C} 1-\mathrm{C} 11$ & $119.6(2)$ \\
\hline $\mathrm{O} 1-\mathrm{C} 8-\mathrm{C} 9$ & $125.2(2)$ & $\mathrm{C} 6-\mathrm{C} 1-\mathrm{Cl1}$ & $119.8(3)$ \\
\hline $\mathrm{N} 2-\mathrm{C} 8-\mathrm{C} 9$ & $114.5(2)$ & $\mathrm{C} 3-\mathrm{C} 4-\mathrm{C} 5$ & $120.9(3)$ \\
\hline $\mathrm{C} 6-\mathrm{C} 5-\mathrm{C} 4$ & $118.5(2)$ & $\mathrm{C} 3-\mathrm{C} 4-\mathrm{H} 4$ & 119.6 \\
\hline
\end{tabular}




$\begin{array}{ll}\mathrm{C} 6-\mathrm{C} 5-\mathrm{C} 7 & 121.1(3) \\ \mathrm{C} 4-\mathrm{C} 5-\mathrm{C} 7 & 120.4(2) \\ \mathrm{C} 12-\mathrm{C} 17-\mathrm{C} 16 & 120.2(2) \\ \mathrm{C} 12-\mathrm{C} 17-\mathrm{H} 17 & 119.9 \\ \mathrm{C} 16-\mathrm{C} 17-\mathrm{H} 17 & 119.9 \\ \mathrm{~N} 2-\mathrm{C} 18-\mathrm{C} 19 & 112.24(19) \\ \mathrm{N} 2-\mathrm{C} 18-\mathrm{H} 18 \mathrm{~A} & 109.2 \\ \mathrm{C} 19-\mathrm{C} 18-\mathrm{H} 18 \mathrm{~A} & 109.2 \\ \mathrm{~N} 2-\mathrm{C} 18-\mathrm{H} 18 \mathrm{~B} & 109.2 \\ \mathrm{C} 19-\mathrm{C} 18-\mathrm{H} 18 \mathrm{~B} & 109.2 \\ \mathrm{H} 18 \mathrm{~A}-\mathrm{C} 18-\mathrm{H} 18 \mathrm{~B} & 107.9 \\ \mathrm{C} 15-\mathrm{C} 14-\mathrm{C} 13 & 119.8(2) \\ \mathrm{C} 15-\mathrm{C} 14-\mathrm{H} 14 & 120.1\end{array}$

$\begin{array}{ll}\mathrm{C} 5-\mathrm{C} 4-\mathrm{H} 4 & 119.6 \\ \mathrm{C} 1-\mathrm{C} 2-\mathrm{C} 3 & 119.7(3) \\ \mathrm{C} 1-\mathrm{C} 2-\mathrm{H} 2 & 120.1 \\ \mathrm{C} 3-\mathrm{C} 2-\mathrm{H} 2 & 120.1 \\ \mathrm{C} 2-\mathrm{C} 3-\mathrm{C} 4 & 120.3(3) \\ \mathrm{C} 2-\mathrm{C} 3-\mathrm{H} 3 & 119.9 \\ \mathrm{C} 4-\mathrm{C} 3-\mathrm{H} 3 & 119.9 \\ \mathrm{C} 20-\mathrm{C} 21-\mathrm{H} 21 \mathrm{~A} & 109.5 \\ \mathrm{C} 20-\mathrm{C} 21-\mathrm{H} 21 \mathrm{~B} & 109.5 \\ \mathrm{H} 21 \mathrm{~A}-\mathrm{C} 21-\mathrm{H} 21 \mathrm{~B} & 109.5 \\ \mathrm{C} 20-\mathrm{C} 21-\mathrm{H} 21 \mathrm{C} & 109.5 \\ \mathrm{H} 21 \mathrm{~A}-\mathrm{C} 21-\mathrm{H} 21 \mathrm{C} & 109.5 \\ \mathrm{H} 21 \mathrm{~B}-\mathrm{C} 21-\mathrm{H} 21 \mathrm{C} & 109.5\end{array}$

Hydrogen-bond geometry $(\AA, \stackrel{\circ}{)})$

$\mathrm{Cg} 2$ is the centroid of the $\mathrm{C} 1-\mathrm{C} 6$ phenyl ring

\begin{tabular}{lllll}
\hline$D-\mathrm{H} \cdots A$ & $D-\mathrm{H}$ & $\mathrm{H} \cdots A$ & $D \cdots A$ & $D-\mathrm{H} \cdots A$ \\
\hline $\mathrm{C} 14-\mathrm{H} 14 \cdots \mathrm{O} 2^{\mathrm{i}}$ & 0.93 & 2.53 & $3.416(3)$ & 160 \\
$\mathrm{C} 7-\mathrm{H} 7 B \cdots \mathrm{O} 1^{\mathrm{ii}}$ & 0.97 & 2.54 & $3.485(3)$ & 164 \\
$\mathrm{C} 15-\mathrm{H} 15 \cdots \mathrm{O} 1^{\mathrm{iii}}$ & 0.93 & 2.66 & $3.474(3)$ & 147 \\
$\mathrm{C} 20-\mathrm{H} 20 B \cdots C g 2^{\text {iv }}$ & 0.97 & 2.81 & $3.759(3)$ & 165
\end{tabular}

Symmetry codes: (i) $-x,-y+1,-z+1$; (ii) $-x+1,-y+2,-z+1$; (iii) $x-1, y-1, z$; (iv) $-x+1,-y,-z+1$. 